\title{
EXPERIMENTAL TESTS OF THE GDH AND OTHER SUM RULES AT SLAC
}

\author{
P. E. BOSTED \\ University of Massachusetts, Amherst, Massachusetts 01003
}

\begin{abstract}
Recent measurements from SLAC of the polarized nucleon structure functions $g_{1}$ and $g_{2}$ have been used to experimentally test the Bjorken, Ellis-Jaffe, BurkhardtCottingham, and Efremov-Leader-Teryaev sum rules. In the future, the SLAC E159 experiment will extend sturcture function measurements using real photons to $40 \mathrm{GeV}$, enabling a definitive test of the high energy convergence of the GDH sum rule for both proton and deuteron targets.
\end{abstract}

\section{Sum Rules involving $g_{1}$}

The fundemental Bjorken sum rule ${ }^{1}$ rigorously relates the integral of the difference of proton and neutron polairzed structure functions $g_{1}^{p}(x)$ and $g_{1}^{n}(x)$ to $g_{A} / g_{V}$, measured in neutron beta decay. The sum rule is derived using current algebra, and strickly speaking is valid in the limit of infinite $Q^{2}$, although pQCD allows radiative corrections to be estimated accurately. It is of particular interest to this conference on the GDH sum rule, which is essentailly the $Q^{2}=0$ limit of the Bjorken sum rule, dervied using an unsubtracted dispersion relation, and valid for both protons and neutrons individually.

A recent experiment ${ }^{2}$ at SLAC, E155, has made improved measurements of the $g_{1}$ structure function using both polarized proton and deuteron targets. The E155 collaboration made an NLO pQCD fit to the world data set (dominated statistically at moderate and high $x$ by the E155 and E143 data), to evaluate the Bjorken sum rule at $Q^{2}=5 \mathrm{GeV}^{2}$. The result, $\int_{0}^{1}\left[g_{1}^{p}(x)-g_{1}^{n}(x)\right] d x=0.176 \pm 0.003$ (stat) \pm 0.007 (syst), is in good agreement with the Bjorken sum rule prediction of $0.182 \pm 0.005$ evaluated with up to third order corrections ${ }^{3}$ in $\alpha_{s}$. The largest uncertainty in the experimental evaluation comes from the extrapolation to $x=0$.

On the other hand, the E155 fit finds the quark singlet contribution $\Delta \Sigma=0.23 \pm 0.04$ (stat) \pm 0.06 (syst), well below the Ellis-Jaffe sum rule prediction $^{4}$ of 0.58 . However, this well-known result (originally dubbed 
the "spin crisis") now has several possible explanations, arising from the several assumptions made in the derivation of the Ellis-Jaffe sum rule. For example, the strange quark contribution may not be zero, or the gluon spin could be substantial.

\section{Sum rules involgin $g_{2}$}

The E155x collaboration at SLAC recently submitted for publication ${ }^{5}$ new results for the $g_{2}$ polarized structure function with errors typically three times smaller than previous data. This permits two interesting sum rules to be tested. The Burkhardt-Cottingham sum rule ${ }^{6}$ for $g_{2}$ at large $Q^{2}, \int_{0}^{1} g_{2}(x) d x=0$, was derived from virtual Compton scattering dispersion relations. It does not follow from the Operator Product Expansion. Its validity depends on the lack of singularities for $g_{2}$ at $x=0$, and a dramatic rise of $g_{2}$ at low $x$ could invalidate the sum rule ${ }^{7}$ E155x evaluated the Burkhardt-Cottingham integral in the measured region of $0.02 \leq x \leq 0.8$ at $Q^{2}=5 \mathrm{GeV}^{2}$. The results for the proton and deuteron are $-0.044 \pm 0.008 \pm 0.003$ and $-0.008 \pm 0.012 \pm 0.002$ respectively. This does not represent a conclusive test of the sum rule because the behavior of $g_{2}$ as $x \rightarrow 0$ is not known. However, if we assume that $g_{2}$ equlas the twist2 Wandzura-Wilczek ${ }^{8}$ expression $g_{2}^{W W}$ for $x<0.02$, and use the relation $\int_{0}^{x} g_{2}^{W W}(y) d y=x\left[g_{2}^{W W}(x)+g_{1}(x)\right]$, there is an additional contribution of 0.020 (0.004) for the proton (deuteron), which reduces the disagreement between experiment and prediction. However, a tantalizing $3 \sigma$ discrepency would still remain for the proton.

The Efremov-Leader-Teryaev (ELT) sum rule ${ }^{9}$ involves the valence quark contributions to $g_{1}$ and $g_{2}: \int_{0}^{1} x\left[g_{1}^{V}(x)+2 g_{2}^{V}(x)\right] d x=0$. Assuming that the sea quarks are the same in protons and neutrons, the sum rule takes a form $\int_{0}^{1} x\left[g_{1}^{p}(x)+2 g_{2}^{p}(x)-g_{1}^{n}(x)-2 g_{2}^{n}(x)\right] d x=0$. E155x evaluated this ELT integral in the measured region using the E155x $g_{2}$ data and the E155 fit to $g_{1}$. The result at $Q^{2}=5 \mathrm{GeV}^{2}$ is $-0.013 \pm 0.008 \pm 0.002$, which is consistent with the expected value of zero. The extrapolation to $x=0$ is not known, but is suppressed by a factor of $x$, so is not expected to make much difference.

In summary, the more rigourous Bjorken and ELT sum rules are verified experimentally, but the assumptions going into the Ellis-Jaffe sum rule are clearly invalidated. The situation with the BC sum rule is less clear, but the data hint at the need for substantial contributions at low $x$ beyond the twist-2 Wandzura-Wilczek terms, or a break-down of the assumption 
that an unsubtracted dispersion relation can be used. This of particular relevance to the GDH sum rule, which relies on a similar assumption. A conclusive experimental meassurement of the GDH sum rule will require photon energies well above $5 \mathrm{GeV}$. For this reason, the SLAC Real Photon Collaboration plans to perform an experiment ${ }^{10}$ (E159) up to $40 \mathrm{GeV}$ photon energies in the next few years.

\section{Proposal to Measure $\Delta \sigma^{\gamma N}(k)$ and the High Energy Contribution to the GDH Sum Rule}

The E159 experiment will use an untagged coherent bremsstrahlung beam to create a high flux of circularly polarized photons. With coherent bremsstrahlung, a set of high intensity spikes is generated by proper orientation of a diamond crystal radiator ${ }^{11}$. Some typical intensity spectra for E159 are shown in Fig. 1. A good way to check systematics is to run with similar coherent peak energies but two different electron energies. This is illustrated in cases b) and c) in Fig. 1

With longitudinally polarized electrons, the incoherent bremsstrahlung photons are circularly polarized according to $P_{\gamma}=P_{e} y(4-y) /\left(4-4 y+3 y^{2}\right)$, where $y=k / E$ is the ratio of photon and electron energies, and $P_{\gamma}$ and $P_{e}$ are the photon and electron polarizations. The coherent photons are elliptically polarized: the circular component is almost identical to that for incoherent photons ${ }^{12}$, providing a high degree of circular polarization (relative to the electron polarization) for $k / E>0.5$, the minimum value E159. This is illustrated in Fig. 2. The coherent peak polarization also has a linear component which will cancel in the measurement of $\Delta \sigma^{\gamma N}(k)$ (since the orientation does not change sign when the electron helicity is flipped on a pulse-to-pulse basis), while the circular component does. The effect of linear polarization also cancels in the measurement of the total cross section $\sigma^{\gamma N}(k)$ because the detector system is azimuthally symmetric.

A low-Z beam hardener may be used to reduce the intensity of photons below $100 \mathrm{MeV}$. The flux of low energy photons will also be reduced due to the LPM effect. A collimator will be used to enhance the ratio of coherent to incoherent radiation, as was done in a previous SLAC experiment ${ }^{13}$. For photon energies up to about $30 \mathrm{GeV}$, a substantial fraction of the measured $\Delta \sigma^{\gamma N}(k)$ will come from the primary intensity spike. The contributions from low energy, incoherent photons will be measured using an amorphous carbon radiator. A simultaneous fit to all the measurements will produce a best fit for $\Delta \sigma^{\gamma N}$ as a function of photon energy $k$. 

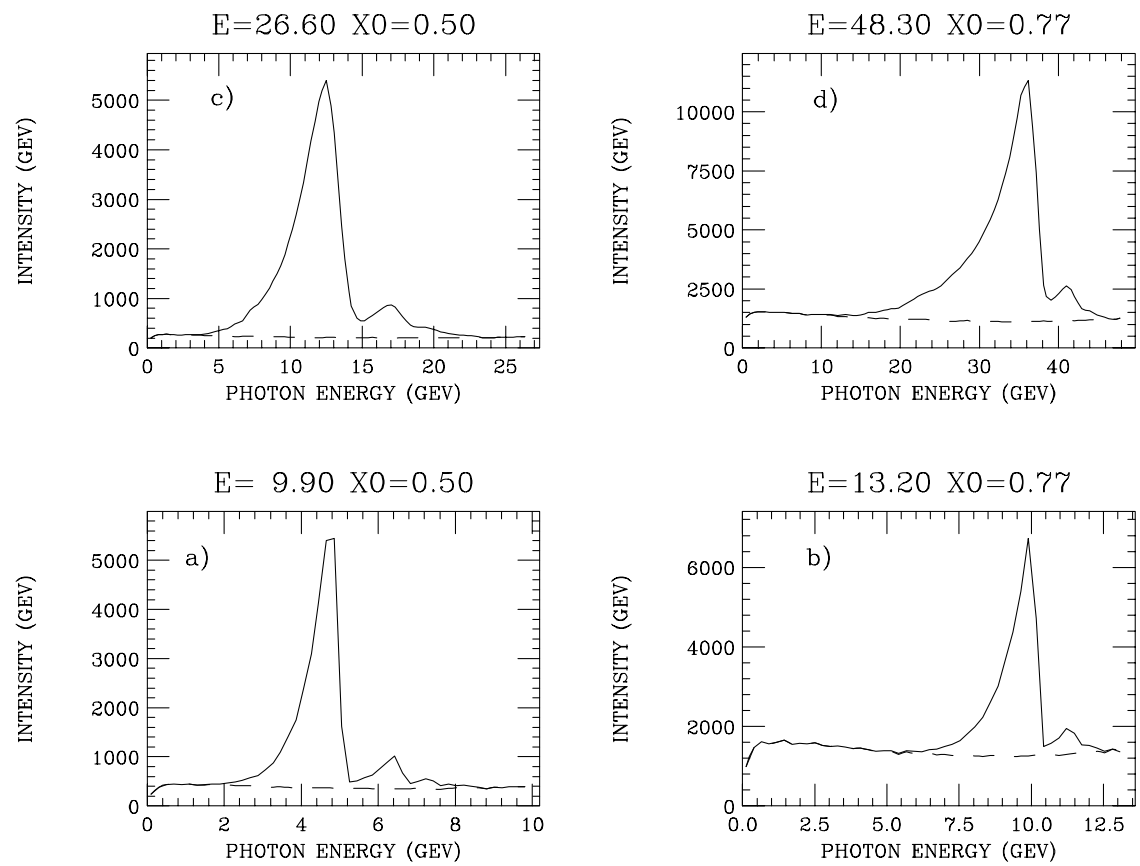

Figure 1. Calculated intensity (flux times energy) for collimated coherent bremsstrahlung at four settings for E159. The dashed lines are incoherent radiation only, while the solid lines include coherent contributions.
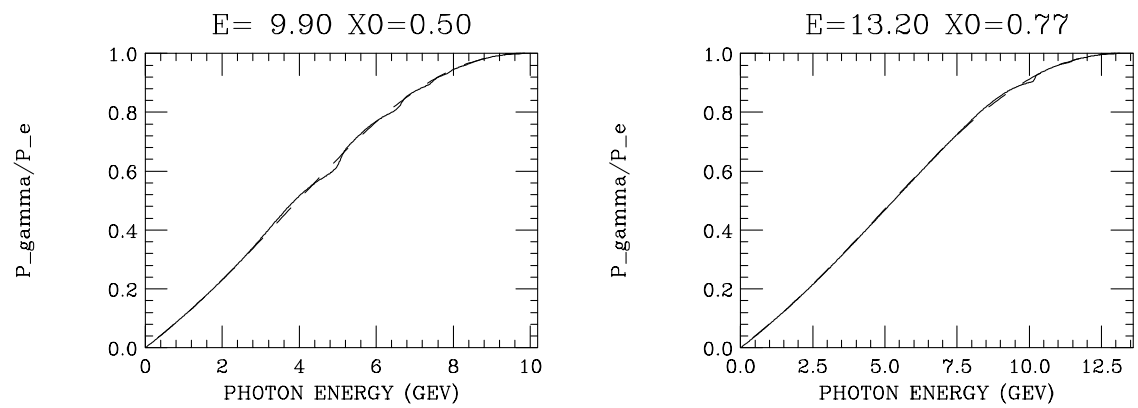

Figure 2. Photon circular polarization (relative to electron polarization) as a function of photon energy for the spectra of Fig. 1a and 1b. The dashed lines are for incoherent radiation while the solid lines include the coherent peaks.

For targets, E159 will use polarized $\mathrm{NH}_{3}$ and $\mathrm{ND}_{3}$ as sources of polarized protons and neutrons. Polarized deuterons to first order allow measurements of the isovector combination $(n+p) / 2$, with small corrections for the 
deuteron $D$-state, shadowing, and nuclear coherent hadron production. An extension to E159 could use a polarized ${ }^{3} \mathrm{He}$ target to verify the consistency of $\Delta \sigma^{\gamma n}(k)$ for the neutron as extracted from either deuterium or ${ }^{3} \mathrm{He}$. The detector is optimized to measure $>98 \%$ of all hadronic interactions, and to reject electromagnetic backgrounds.

E159 plans to take data in two modes: counting mode and flux integration mode. In the counting mode, all particles except neutrinos emerging from the target with sufficient energy and angle $\theta_{\min }<\theta<\theta_{\max }$ are counted in one of two total absorption lead/scintillator calorimeters, with each segmented longitudinally for electromagnetic showers (the first 27 r.l.) and hadronic showers (the remaining 53 r.l.). A schematic layout of the target and detector system is shown in Fig. 3. A hadronic interaction is identified with very high efficiency using suitable kinematic cuts that reduce the electromagnetic background to a small level. The target geometry fixes $\theta_{\max }$ at 40 degrees, while the movable detector geometry determines $\theta_{\min }=0.01 \sqrt{40 / k_{0}}$ as an optimal tradeoff between signal and background. Approximately five hadronic interactions originating from coherent photons will be detected per beam spill in the counting mode.

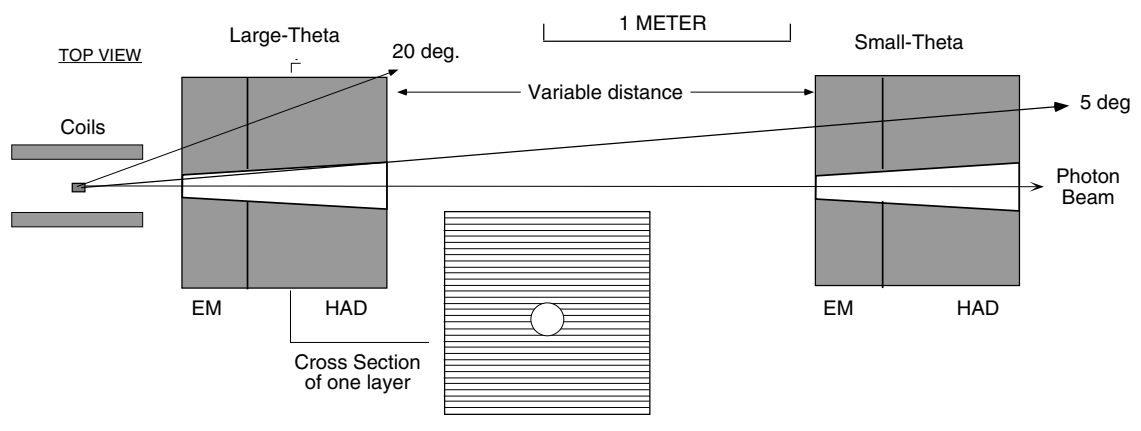

Figure 3. Two views of the E159 detector package in relation to the polarized target.

Using the counting mode, E159 should be able to verify the reasonable assumptions that the energy per event in the hadronic part of the calorimeter is independent of photon helicity, and that the total energy from electromagnetic background events is negligible. If these assumptions are verified, E159 can obtain significantly smaller statistical errors by raising the beam current to a level corresponding to several hundred nuclear interactions per beam spill, and simply measuring the asymmetry in the total energy deposition in the hadronic part of the calorimeter. As a further systematic 
check, E159 can also integrate the energy in both the electromagnetic and hadronic portions of the detector, and use calculations to account for the rate and asymmetry of the electromagnetic processes ${ }^{14,15}$. Approximately one-half of all the energy in the calorimeters comes from electromagnetic processes, with a very small asymmetry expected. This method has the advantage of minimizing the sensitivity to a possible helicity-dependence to the fractional energy into $\pi^{0}$ 's.

E159 plans to extract $\Delta \sigma^{\gamma N}$ from the data in two ways. In the first method, the difference of rates for the two helicity states is converted to cross sections directly using the measured detector acceptance and target thickness. In the second method, the asymmetry in counting rates will be corrected by a calculated dilution factor, and converted to $\Delta \sigma^{\gamma N}$ using previously measured values of $\sigma^{\gamma N}(k)$. In this method, factors such as the detector efficiency cancel, so that some systematic errors will be reduced (while others will be increased). The expected magnitude of the experimental asymmetries is relatively small, and will likely be less than 0.01 at the lowest photon beam energy, and a factor of 10 to 100 smaller at higher energies. Comparison of the two analysis methods will give a valuable check on the evaluation of systematic errors.

The expected errors are shown in Fig. 4 for both the proton and neutron, and for the counting mode and flux integration mode. Even with the larger counting mode statistical errors, a very good determination can be made of both the magnitude and energy dependence of $\Delta \sigma^{\gamma N}(k)$ for $5<k<40$ $\mathrm{GeV}$. By measuring with both proton and deuteron targets, the high energy contributions to both the isovector and isoscalar GDH sum rules can be determined. This will allow tests of Regge-inspired models, which predict very different behavior for the isovector and isoscalar contributions, and will provide a baseline for studies of the polarized spin-structure functions measured with virtual photons. Surprises are also possible: the data may not turn out to look like any of the Regge models at all. In any case, E159

will provide the definitive measurements needed to determine the energy scale of convergence of the GDH sum rule, and will provide a quantitiative tet for its magnitude for both the proton and the deuteron.

\section{Acknowledgments}

Work supported by the National Science Foundation and the Department of Energy contract DE-AC03-76SF00515. 

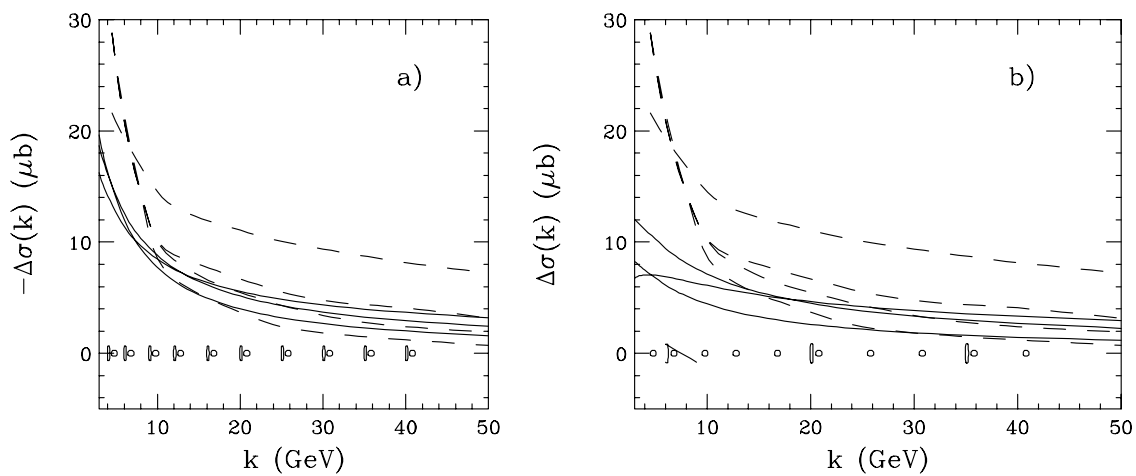

Figure 4. a) Estimated proton error bars for $\Delta \sigma^{\gamma p}(k)$ for this proposal as a function of photon energy for the counting mode (rectangles) and flux integration mode (circles). The dashed curves are representative models from Ref. 16, the solid curves are from Ref. 17 ; b) same but for the neutron as measured with $\mathrm{ND}_{3}$.

\section{References}

1. J. D. Bjorken, Phys. Rev. 148, 1467 (1966) ; Phys. Rev. D1, 1376 (1970).

2. E155 Collaboration, Phys. Lett. B463, 339 (1999); Phys. Lett. B493, 19 (1999).

3. S. A. Larin and J. A. M. Vermaseren, Phys. Lett. B259, 345 (1991) and references therein.

4. J. Ellis and R. Jaffe, Phys. Rev. D9, 1444 (1974); D10, 1669 (1974) (E).

5. E155x Collaboration, SLAC-PUB-8813, hep-ex/0204028, submitted to Phys. Lett. (2002).

6. H. Burkhardt and W. N. Cottingham, Ann. Phys. 56, 453 (1970).

7. I. P. Ivanov et al., Phys. Rep. 320, 175 (1999).

8. S. Wandzura and F. Wilczek, Phys. Lett. B72, 195 (1977).

9. A. V. Efremov, O. V. Teryaev and E. Leader, Phys. Rev. D55, 4307 (1997).

10. http://www.slac.stanford.edu/exp/e159

11. G. Diambrini Palazzi, Rev. Mod. Phys. 40, 611 (1968).

12. I. M. Nadzhafov, Bulletin of the Academay of Sciences of the USSR, Physical Series Vol 14, No. 10, p. 2248 (1976).

13. W. Kaune et al., Phys. Rev. D11, 478 (1975).

14. Y.S. Tsai, Rev. Mod. Phys. 46, 815 (1974); 49, 421 (1977) (E).

15. T. Gehrmann, M. Stratmann, Phys. Rev. D56, 5839 (1997).

16. S. D. Bass and M. M. Brisudova, Eur. Phys. J. A4, 251 (1999); S. D. Bass, Mod. Phys. Lett. A12, 1051 (1997) and references therein.

17. N. Bianchi and E. Thomas, Phys. Lett. B450, 439 (1999); E. Thomas and N. Bianchi, Nucl. Phys. Proc. Suppl. 82, 256 (2000). 